\title{
Inflation with non-canonical scalar fields revisited
}

\author{
Smaragda Lola ${ }^{1, \mathrm{a}}$, Andreas Lymperis ${ }^{1, \mathrm{~b}}$, Emmanuel N. Saridakis ${ }^{2,3,4, \mathrm{c}}$ \\ ${ }^{1}$ Department of Physics, University of Patras, 26500 Patras, Greece \\ 2 National Observatory of Athens, Lofos Nymfon, 11852 Athens, Greece \\ ${ }^{3}$ Department of Physics, National Technical University of Athens, Zografou Campus, 15773 Athens, Greece \\ ${ }^{4}$ Department of Astronomy, School of Physical Sciences, University of Science and Technology \\ of China, Hefei 230026, People's Republic of China
}

Received: 31 March 2021 / Accepted: 30 July 2021 / Published online: 10 August 2021

(C) The Author(s) 2021

\begin{abstract}
We revisit inflation with non-canonical scalar fields by applying deformed-steepness exponential potentials. We show that the resulting scenario can lead to inflationary observables, and in particular to scalar spectral index and tensor-to-scalar ratio, in remarkable agreement with observations. Additionally, a significant advantage of the scenario is that the required parameter values, such as the noncanonicality exponent and scale, as well as the potential exponent and scale, do not need to acquire unnatural values and hence can accept a theoretical justification. Hence, we obtain a significant improvement with respect to alternative schemes, and we present distinct correlations between the model parameters that better fit the data, which can be tested in future probes. This combination of observational efficiency and theoretical justification makes the scenario at hand a good candidate for the description of inflation.
\end{abstract}

\section{Contents}

1 Introduction . . . . . . . . . . . . . . 1

2 Non-canonical inflation with deformed-steepness

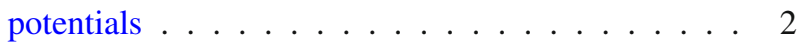

3 Results .................. . . 3

4 Conclusions . . . . . . . . . . . . 7

References................... 8

\section{Introduction}

Inflation is now a crucial part of the Standard Model of Cosmology [1-5]. Its solution to the horizon and flatness

\footnotetext{
a e-mail: magda.lola@upatras.gr

b e-mail: alymperis@upatras.gr (corresponding author)

ce-mail:msaridak@phys.uoa.gr
}

problems, together with the predictions for an almost scale invariant perturbation spectral index, have been confirmed by measurements of the cosmic microwave background (CMB) radiation. Nevertheless, the specific mechanism that triggers the inflationary epoch is one of the most outstanding issues in contemporary particle physics and cosmology. As a result, the building of theoretical models that explain this early accelerating expansion of the universe has exploded in recent years. The first main class of mechanisms that can lead to successful inflation is based on the introduction of a scalar field, while the second main class is obtained through gravitational modifications (for reviews see [6-10]). Consequently, inflation-related observations have provided significant insight to both modified gravity [11-16], as well as to particle physics model building. The literature on the latter is very extensive, particularly within the framework of supersymmetry [17-20], supergravity [21-24], theories of extra dimensions such as superstring and brane theories [25-27], and technicolor too [28]. Detailed lists of references on different theoretical constructions can be found in $[6,7,10]$.

In trying to understand the above issues (often in the framework of a single theory) several problems have been encountered, including fine-tuning issues (tiny dimensionless constants) and large predictions for tensor fluctuations. In this respect, theories of scalar fields with non-canonical kinetic terms, as expected in supergravity and superstring theories, including the $k$-inflation subclass [29-31], were found to have significant advantages. These theories arise commonly in the framework of supergravity and string compactifications, which typically contain a large number of light scalar fields $X$ (moduli), whose dynamics are governed by a non-trivial moduli space metric $G_{i j}$. As long as the moduli space metric is not flat, we generically expect non-canonical kinetic terms. Such effects could, but need not, be suppressed by the high scale of the corresponding Ultra-Violet physics (e.g. moduli masses, string scale), but they can still have sig- 
nificant cosmological consequences through the dynamics of the dilaton and moduli fields.

Among their many advantages, non-canonical scalars satisfy in a more efficient way the slow-roll conditions of inflation, since the additional effective friction terms in the equations of motion of the inflaton slow down the scalar field for potentials which would otherwise be too steep. Hence, the resulting tensor-to-scalar ratio is significantly reduced [32-49]. Moreover, models with non-canonical kinetic terms often allow for the kinetic term to play the role of dark matter and the potential terms to generate dark energy and inflation [50-53]. Additionally, note that in the inflation realization in the context of Galileon and Horndeski theories, the role of the non-canonical kinetic term is also crucial [54-60]. The form of the non-canonical terms can vary significantly, since there are many plausible models, including different ways to achieve compactification. The recent cosmological data, however, together with the requirement to avoid fine-tuning and non-physical solutions, severely constrain the available possibilities.

On the other hand, an alternative way to improve the inflationary observables is by introducing an extra parameter as an exponent in the known potential forms, and thus affecting their steepness. In this way the dynamics of the scalar field can be additionally deformed, offering an alternative way to bring the tensor-to-scalar ratio to lower values without ruining the necessary spectral index [61-71].

One possible disadvantage of the above inflationary models, namely those with non-canonical terms and those with extra steepness parameter in the potential, is that the parameter values needed for acceptable observables are quite unnatural (with "natural" meaning the standard values of canonical kinetic term, no extra steepness parameter, and sub-Planckian potential values) and hard to be justified from the fieldtheoretical point of view. In particular, the non-canonical exponents need to be large, or the mass and potential parameters take trans-Planckian values. Hence, in this work, we are interested in studying a combination of the above models, specifically introducing a scalar field with non-canonical kinetic terms on top of a deformed-steepness potential with an extra parameter. As we will show, this enhances the range of solutions and leads to very satisfactory observables, for sets of model parameters significantly closer to the natural ones, that we proceed to identify and classify.

\section{Non-canonical inflation with deformed-steepness potentials}

In this section we present the scenario of non-canonical inflation with deformed-steepness potentials. We will focus on the usual non-canonical Lagrangian, which is well justified theoretically, and takes the form [29-31,36,72,73],

$\mathcal{L}(\phi, X)=X\left(\frac{X}{M^{4}}\right)^{\alpha-1}-V(\phi)$,

where $X=\frac{1}{2} \partial_{\mu} \phi \partial^{\mu} \phi$ is the kinetic energy of the scalar field, and thus the action of the scenario reads

$\mathcal{S}=\int d^{4} x \sqrt{-g}\left[M_{p l}^{2} \frac{R}{2}+X\left(\frac{X}{M^{4}}\right)^{\alpha-1}-V(\phi)\right]$.

The parameter $M$ has dimensions of mass and determines the scale in which the non-canonical effects become significant, while $M_{p l}$ is the Planck mass. Concerning the potential, in this work we will consider the deformed-steepness potential that was introduced in $[61,63]$, namely

$V(\phi)=V_{0} e^{-\lambda \phi^{n} / M_{p l}^{n}}$

with $V_{0}$ and $\lambda$ the usual potential parameters and $n$ the new exponent parameter that determines the deformed-steepness.

We consider a homogeneous and isotropic flat FriedmannRobertson-Walker (FRW) metric of the form

$d s^{2}=-d t^{2}+a^{2}(t) \delta_{i j} d x^{i} d x^{j}$,

where $a(t)$ is the scale factor. Variation of the action (2.2) in terms of the metric gives the following Friedmann equations

$$
\begin{aligned}
H^{2} & =\frac{1}{3 M_{p l}^{2}}\left[(2 \alpha-1) X\left(\frac{X}{M^{4}}\right)^{\alpha-1}+V_{0} e^{-\lambda \phi^{n} / M_{p l}^{n}}\right] \\
\dot{H} & =-\frac{1}{M_{p l}^{2}} \alpha X\left(\frac{X}{M^{4}}\right)^{\alpha-1},
\end{aligned}
$$

where $H=\frac{\dot{a}}{a}$ is the Hubble parameter. Additionally, variation in terms of the scalar field leads to the Klein-Gordon equation

$\ddot{\phi}+\frac{3 H \dot{\phi}}{2 \alpha-1}-\frac{\lambda n \phi^{n-1} V_{0} e^{-\lambda \phi^{n} / M_{p l}^{n}}}{\alpha(2 \alpha-1) M_{p l}^{n}}\left(\frac{2 M^{4}}{\dot{\phi}^{2}}\right)^{\alpha-1}=0$.

Note that one can write the above equation in the form of the usual conservation equation $\dot{\rho}_{\phi}+3 H\left(\rho_{\phi}+p_{\phi}\right)$, using the definitions

$$
\begin{aligned}
& \rho_{\phi}=(2 \alpha-1) X\left(\frac{X}{M^{4}}\right)^{\alpha-1}+V_{0} e^{-\lambda \phi^{n} / M_{p l}^{n}} \\
& p_{\phi}=X\left(\frac{X}{M^{4}}\right)^{\alpha-1}-V_{0} e^{-\lambda \phi^{n} / M_{p l}^{n}} .
\end{aligned}
$$

In every inflationary scenario the important quantities are the inflation-related observables, namely the scalar spectral index of the curvature perturbations $n_{\mathrm{s}}$ and its running $\alpha_{\mathrm{s}} \equiv d n_{\mathrm{s}} / d \ln k$, with $k$ the measure of the wave number $\mathbf{k}$, the tensor spectral index $n_{\mathrm{T}}$ and its running, as well as the 
tensor-to-scalar ratio $r$. In a given scenario these quantities depend on the model parameters, and hence confrontation with observational data can lead to constraints on these model parameters.

In order to extract the relations for the inflation-related observables, a detailed and thorough perturbation analysis is needed. In the simple case of canonical fields minimally coupled to gravity, and introducing the slow-roll parameters, full perturbation analysis indicates that the inflationary observables can be expressed solely in terms of the scalar potential and its derivatives [7,10,74]. However, in the case where non-canonical terms or forms of non-minimally coupling are present, as well as in the case where the potential itself is absent (as for instance in modified gravity inflation), one should instead introduce the Hubble slow-roll parameters $\epsilon_{n}$ (with $n$ positive integer), defined as [10,75-77]

$\epsilon_{n+1} \equiv \frac{d \ln \left|\epsilon_{n}\right|}{d N}$

where $N \equiv \ln \left(a / a_{i n i}\right)$ is the e-folding number, and $\epsilon_{0} \equiv$ $H_{i n i} / H$, where $a_{i n i}$ is the initial scale factor with $H_{i n i}$ the corresponding Hubble parameter (as usual inflation ends when $\epsilon_{1}=1$ ). Thus, the first three $\epsilon_{n}$ are found to be

$$
\begin{aligned}
\epsilon_{1} \equiv & -\frac{\dot{H}}{H^{2}}, \\
\epsilon_{2} \equiv & \frac{\ddot{H}}{H \dot{H}}-\frac{2 \dot{H}}{H^{2}}, \\
\epsilon_{3} \equiv & \left(\ddot{H} H-2 \dot{H}^{2}\right)^{-1} \\
& \times\left[\frac{H \dot{H} \dddot{H}-\ddot{H}\left(\dot{H}^{2}+H \ddot{H}\right)}{H \dot{H}}-\frac{2 \dot{H}}{H^{2}}\left(H \ddot{H}-2 \dot{H}^{2}\right)\right] .
\end{aligned}
$$

With these definitions, the basic inflationary observables are given as [10]

$$
\begin{aligned}
r & \approx 16 c_{s} \epsilon_{1}, \\
n_{\mathrm{s}} & \approx 1-2 \epsilon_{1}-\epsilon_{2}, \\
\alpha_{\mathrm{S}} & \approx-2 \epsilon_{1} \epsilon_{2}-\epsilon_{2} \epsilon_{3}, \\
n_{\mathrm{T}} & \approx-2 \epsilon_{1},
\end{aligned}
$$

where the sound speed is defined as [36]

$c_{s}^{2}=\left[\frac{(\partial \mathcal{L} / \partial X)}{(\partial \mathcal{L} / \partial X)+(2 X)\left(\partial^{2} \mathcal{L} / \partial X^{2}\right)}\right]$,

which for the Lagrangian (2.1) gives

$c_{s}^{2}=\frac{1}{2 \alpha-1}$.

In the scenario of non-canonical inflation with deformedsteepness potentials, described by equations (2.5)-(2.7), the dynamics, i.e. the Hubble function, is determined by the parameters $\alpha$ and $M$ related to "non-canonicality", by the standard potential parameters $V_{0}$ and $\lambda$, alongside the deformed-steepness parameter $n$. Hence, we deduce that the above inflationary observables (2.13)-(2.16) will be determined by these model parameters too. Nevertheless, we should mention that these parameters are not independent since they are related through the observed value of the amplitude of scalar perturbation $\mathcal{A}_{S}$. In particular, for the action (2.2) one finds [36]

$\mathcal{P}_{S}=\left(\frac{1}{72 \pi^{2} c_{s}}\right)\left\{M_{p l}^{-2(5 \alpha-6)}\left[\frac{\alpha 6^{\alpha}}{M^{4(\alpha-1)}}\right]\left[\frac{V(\phi)^{5 \alpha-2}}{V^{\prime}(\phi)^{2 \alpha}}\right]\right\}^{\frac{1}{2 \alpha-1}}$,

which for our potential (2.3) leads to

$\mathcal{P}_{S}=\mathcal{A}_{S}\left[e^{-(3 \alpha-2) \lambda \frac{\phi^{n}}{M_{p l}^{n}}}\left(\frac{\phi}{M_{p l}}\right)^{2 \alpha(1-n)}\right]^{\frac{1}{2 \alpha-1}}$,

with

$\mathcal{A}_{S}=\left(\frac{1}{72 \pi^{2} c_{s}}\right)\left\{M_{p l}^{4(1-2 \alpha)}\left[\frac{\alpha 6^{\alpha}}{M^{4(\alpha-1)}}\right]\left[\frac{V_{0}^{3 \alpha-2}}{(\lambda n)^{2 \alpha}}\right]\right\}^{\frac{1}{2 \alpha-1}}$.

Since we know that Planck observations give [78]

$\mathcal{A}_{\text {Sobs }} \simeq 2.09052 \times 10^{-9}$,

in the following analysis we set $V_{0}$ at will while $\lambda$ arises from the satisfaction of the above observational constraint.

In the next section we investigate in detail the effect of each parameter on the inflationary observables, and we will show which combinations can bring the predictions deep inside the observational contours.

\section{Results}

In this section, we investigate the inflationary observables in the scenario of non-canonical inflation with deformedsteepness potentials. In particular, we desire to see how the scalar spectral index $n_{\mathrm{s}}$ and the tensor-to-scalar ratio $r$ are affected by the model parameters. Since the involved equations (2.5)-(2.7), the slow-roll parameters (2.10)-(2.12) and the observables expressions (2.13)-(2.16) are in general too complicated to admit analytical solutions, we investigate them numerically.

Specifically, for a given set of parameter values we impose the conditions for $\phi, \dot{\phi}$ and $H$ corresponding to $\epsilon_{i} \ll 1$. According to (2.10)-(2.12), such suitably "small" $\epsilon_{i}$ imply $H \sim$ const. and thus almost exponential expansion, which is the typical feature of inflation. We evolve the system and we determine the end of inflation by demanding at least one of the $\epsilon_{i}$ to become 1 (cases of eternal inflation, in which all 

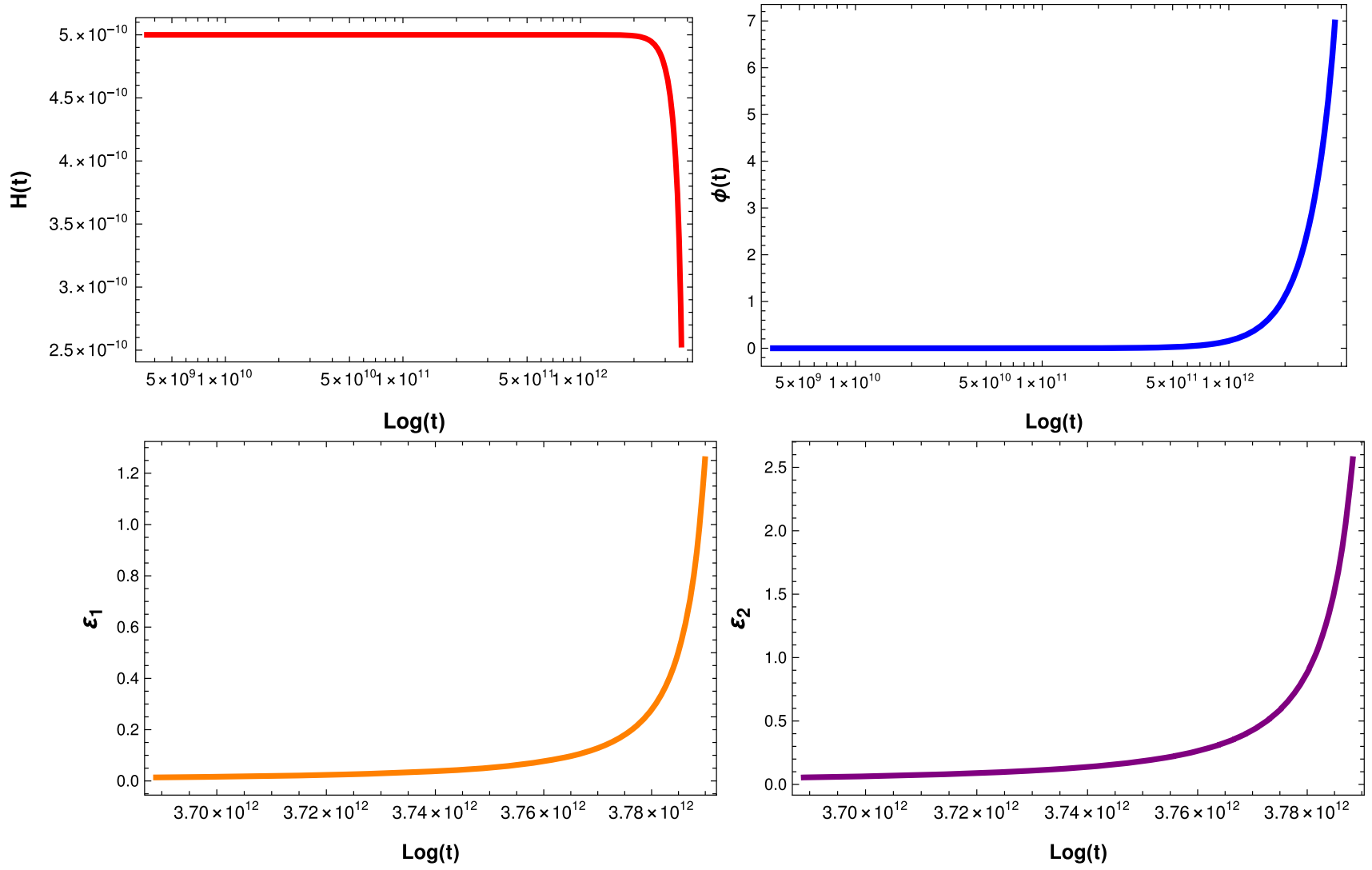

Fig. 1 Time evolution of the Hubble parameter, of the scalar field $\phi$, and of the Hubble slow-roll parameters $\epsilon_{1}$ and $\epsilon_{2}$ in the case where $\alpha=2$ and $n=3$. Initially, and for a suitable time interval, $\epsilon_{i} \ll 1$ and thus according to (2.10)-(2.12) $H$ is almost constant, which corre-

sponds to inflation realization, while as time passes $\epsilon_{i}$ increase and when one of them reaches 1 exponential expansion is not the case anymore ( $H$ decreases) and inflation ends

$\epsilon_{i}$ remain smaller than 1 are considered non-physical), and thus by imposing the desired e-folding number $N$ we extract the time at the beginning of inflation. Hence, we can use the corresponding Hubble parameter to calculate the inflationary observables corresponding to the given parameter values and the imposed e-folding number $N$.

In order to show this in a more transparent way, in Fig. 1 we depict the evolution of $H, \phi, \epsilon_{1}$ and $\epsilon_{2}$ in terms of time. As we observe, initially and for a suitable time interval, $\epsilon_{i}$ are small and thus $H$ is almost constant, which indeed corresponds to inflation realization, while as time passes, $\epsilon_{i}$ increase and when one of them reaches 1 exponential expansion is not the case anymore ( $H$ decreases) and inflation ends. Hence, the imposed initial conditions have been chosen in order for a successful inflation to occur, which requires two conditions: (i) the initial $\epsilon_{i}$ to be $\epsilon_{i} \ll 1$, and (ii) before one of them reaches 1 and inflation ends to have obtained the required "suitable time interval", namely expansion of 50-70 e-foldings. We mention that such background solutions are quite common for the studied range of the model parameters.

We start our investigation by examining the effect of the non-canonical parameter $\alpha$ and the deformed-steepness parameter $n$. Therefore, we fix $M$ and $V_{0}$ at theoretically motivated values and we calculate $n_{\mathrm{s}}$ and $r$ for various combinations of $\alpha$ and $n$, adjusting suitably only the value of $\lambda$ in order to satisfy the observational constraint (2.22), and for the e-folding number $N$ taking, as usual, the values 50,60 and 70. In Table 1 we summarise the obtained observable predictions. Additionally, in order to present the information in a more transparent way that allows comparison with observational data, in Fig. 2 we depict the results of Table 1 on top of the $1 \sigma$ and $2 \sigma$ contours of the Planck 2018 data [78].

A general observation is that the predictions of the scenario at hand lie well inside the $1 \sigma$ region of the Planck 2018 data, without the need to use large values for the noncanonical parameter $\alpha$ or the deformed-steepness parameter $n$, which was indeed the main motivation behind the present work. Additionally, the predictions of the scenario are better, compared to the simple non-canonical models, as well as to the simple deformed-steepness models.

Concerning the specific features, we find the following: For any given set of model-parameters, increasing the efolding values $N$ leads to increased $n_{\mathrm{S}}$ and decreased $r$, as is usual in the majority of inflationary scenarios. Now, for a 
Table 1 Predictions for the scalar spectral index $n_{\mathrm{s}}$ and the tensor-toscalar ratio $r$ for the scenario of non-canonical inflation with deformedsteepness potential, for various combinations of $\alpha$ and $n$, adjusting the values of $\lambda$ in order to satisfy the observational constraint (2.22), and for e-folding number $N$ equal to 50,60 and 70. For this Table we fix $M=10^{-6} M_{p l}$ and $V_{0}=10^{-16} M_{p l}^{4}$, with $M_{p l}=10^{18} \mathrm{GeV}$, while the value of $\lambda$ is determined through the observational constraint (2.22)

\begin{tabular}{llll}
\hline$\alpha=2, n=3, \lambda=7.54 \cdot 10^{-6}$ & & \\
\hline$N$ & 50 & 60 & 70 \\
$r$ & 0.0445 & 0.0359 & 0.0299 \\
$n_{s}$ & 0.9670 & 0.9723 & 0.9761
\end{tabular}

$\alpha=2, n=4, \lambda=5.65 \cdot 10^{-6}$

\begin{tabular}{llll}
\hline$N$ & 50 & 60 & 70 \\
$r$ & 0.0341 & 0.0263 & 0.0209 \\
$n_{s}$ & 0.9649 & 0.9700 & 0.9736
\end{tabular}

$$
\alpha=2, n=5, \lambda=4.52 \cdot 10^{-6}
$$

\begin{tabular}{llll}
\hline$N$ & 50 & 60 & 70 \\
$r$ & 0.0240 & 0.0174 & 0.0130 \\
$n_{s}$ & 0.9611 & 0.9659 & 0.9693 \\
\hline$\alpha=4, n=3, \lambda=7.61 \cdot 10^{-6}$ & & \\
\hline$N$ & 50 & 60 & 70 \\
$r$ & 0.0350 & 0.0288 & 0.0244 \\
$n_{s}$ & 0.9670 & 0.9724 & 0.9763 \\
\hline
\end{tabular}

$\alpha=4, n=4, \lambda=5.71 \cdot 10^{-6}$

\begin{tabular}{llll}
\hline$N$ & 50 & 60 & 70 \\
$r$ & 0.0318 & 0.0257 & 0.0214 \\
$n_{s}$ & 0.9666 & 0.9719 & 0.9758 \\
\hline$\alpha=4, n=5, \lambda=4.57 \cdot 10^{-6}$ & & \\
\hline$N$ & 50 & 60 & 70 \\
$r$ & 0.0282 & 0.0224 & 0.0183 \\
$n_{s}$ & 0.9657 & 0.9710 & 0.9748 \\
\hline$\alpha=6, n=3, \lambda=8.25 \cdot 10^{-6}$ & & \\
\hline$N$ & 50 & 60 & 70 \\
$r$ & 0.0289 & 0.0238 & 0.0202 \\
$n_{s}$ & 0.9668 & 0.9724 & 0.9762 \\
\hline$\alpha=6, n=4, \lambda=6.19 \cdot 10^{-6}$ & & \\
\hline$N$ & 50 & 60 & 70 \\
$r$ & 0.0268 & 0.0218 & 0.0183 \\
$n_{s}$ & 0.9660 & 0.9700 & 0.9759 \\
\hline$\alpha=6, n=5, \lambda=4.95 \cdot 10^{-6}$ & & 0.9746 \\
\hline$N$ & 50 & 60 & \\
$r$ & 0.0245 & 0.0197 & 0.9714 \\
$n_{s}$ & & \\
\hline
\end{tabular}

given $\alpha$, as $n$ increases both $n_{\mathrm{s}}$ and $r$ also increase. On the other hand, for a given $n$, as $\alpha$ increases there is no particular tendency for $n_{\mathrm{s}}$ and $r$. However, for larger $\alpha$, such as $\alpha=6$, the effect of $n$ is less significant and the different curves actually coincide. Moreover, as $\alpha$ grows, $\lambda$ slightly increases, while as $n$ grows $\lambda$ slightly decreases.

We proceed by investigating the effect on the observables of the parameters $M$ and $V_{0}$, which determine the scale of non-canonicality and of the potential, respectively, keeping in mind that the scale of inflation in theoretically motivated constructions can be anywhere from just below the unification scale (mostly Grand Unification), to energies as low as the scales within reach of the LHC (see e.g. [79]), with many possibilities in between, that can be linked i.e. to different stages of symmetry breaking. Without loss of generality we fix $\alpha=2$ and $n=4$ and we calculate $n_{\mathrm{s}}$ and $r$ for e-folding number $N$ being as usual 50,60 and 70. We first additionally fix $M$ and change $V_{0}$, and then we fix $V_{0}$ and change $M$. In all cases we adjust the value of $\lambda$ in order to satisfy the observational constraint (2.22). We summarise the obtained observable predictions in Table 2. Moreover, in order to present the results in a more transparent way, in Fig. 3 we depict the results of Table 2 on top of the $1 \sigma$ and $2 \sigma$ contours of the Planck 2018 data [78].

The main observation is that the predictions of the scenario lie well inside the $1 \sigma$ region of observational data. Now, for fixed $M$, increasing $V_{0}$ leads to lower values of $r$ and $n_{\mathrm{s}}$; moreover, the variation of $r$ is much faster than that of $n_{\mathrm{s}}$. Additionally, for fixed $V_{0}$, increasing $M$ leads also to lower values of $r$ and $n_{\mathrm{s}}$; nevertheless the change in $n_{\mathrm{s}}$ is strongly affected by the change in $M$ (since $M$ appears in powers of four in the equations) that it can easily be led outside the observational contours. This similar tendency behavior was expected, since in the scalar-field equation (2.7) the two parameters $M$ and $V_{0}$ appear multiplied. Nevertheless, this is not a trivial result, since $M$ is related to the non-canonicality scale while $V_{0}$ to the potential scale.

From the above analysis we deduce that non-canonical kinetic terms combined with deformed-steepness potentials can provide inflationary predictions in very good agreement with observations, compared to simple noncanonical models [32-49] as well as to canonical models with deformed-steepness potentials [61-68]. An additional significant advantage is that the above combination allows to achieve good predictions without the need to use unnaturally large values for $\alpha$ or $n$, or unnaturally tuned values for the non-canonicality and potential scales $M$ and $V_{0}$, as well as for the potential exponent $\lambda$. In particular, we see that $M$ and $V_{0}$ remain in reasonable sub-Planckian regions, with values that can be easily predicted and accepted from field theoretical point of view. This combination of observational efficiency and theoretical justification is a significant advantage of the scenario at hand. 

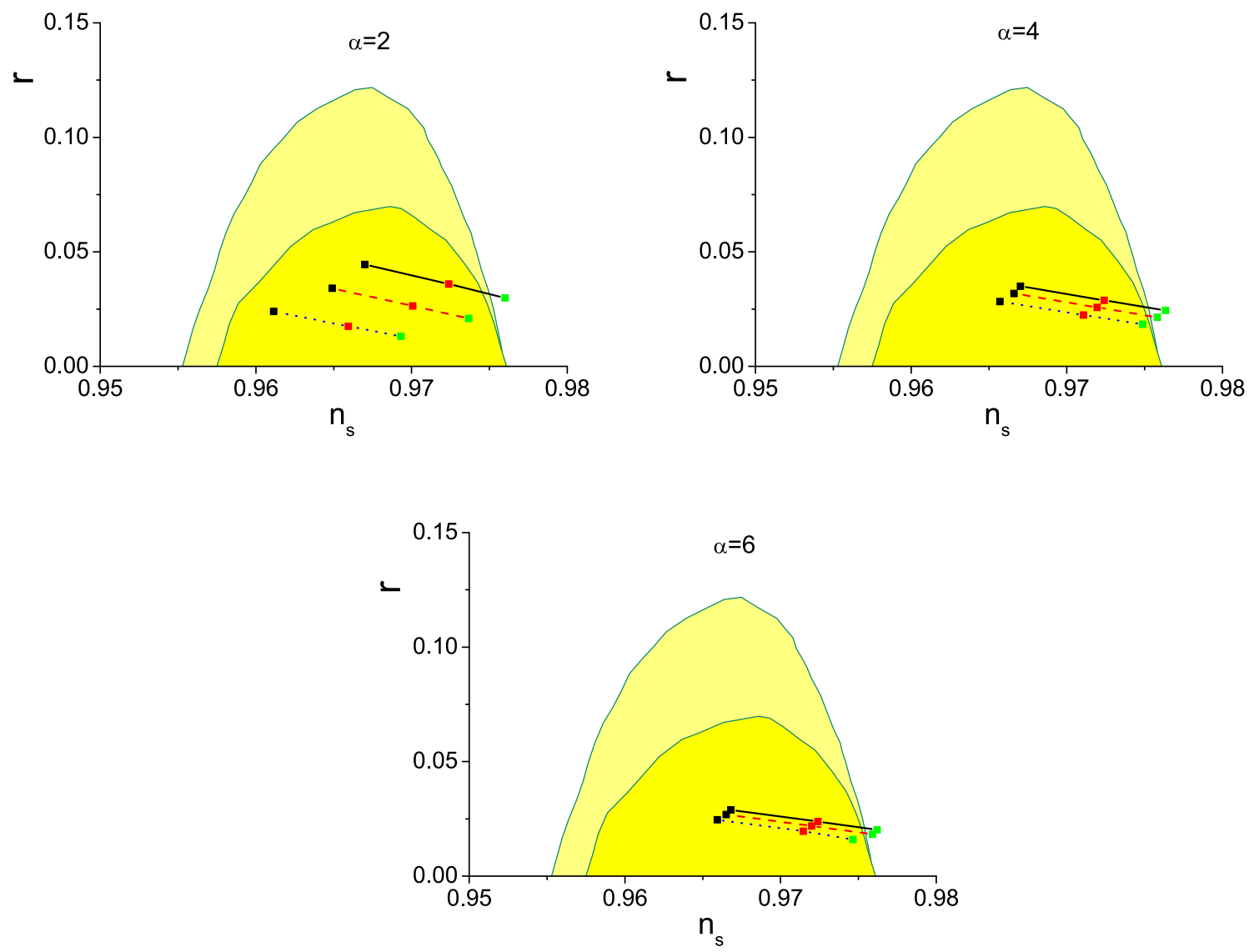

Fig. $21 \sigma$ (yellow) and $2 \sigma$ (light yellow) contours for Planck 2018 results (Planck $+T T+$ low $P$ ) [78], on the $n_{\mathrm{s}}-r$ plane. Furthermore, we depict the predictions of Table 1 , of the scenario at hand for various values of the the non-canonical parameter $\alpha$ and the deformed-steepness parameter $n$, keeping fixed $M=10^{-6} M_{p l}$ and $V_{0}=10^{-16} M_{p l}^{4}$, with $M_{p l}=10^{18} \mathrm{GeV}$, determining the value of $\lambda$ through the observational

Table 2 Predictions for the scalar spectral index $n_{\mathrm{s}}$ and the tensor-toscalar ratio $r$ for the scenario of non-canonical inflation with deformedsteepness potential, for fixed $\alpha=2, n=4$, for e-folding number $N$ being 50, 60 and 70, and with fixed $M$ and varying $V_{0}$ (upper sub- constraint (2.22). In all graphs, the black-solid line corresponds to $n=3$, the red-dashed line to $n=4$ and the blue-dotted line to $n=5$. In every line the first (black) point corresponds to e-folding number $N=50$, the middle (red) point to $N=60$, and the third (green) to $N=70$

Table) and with fixed $V_{0}$ and varying $M$ (lower sub-Table). In all cases the value of $\lambda$ is determined through the observational constraint (2.22), while $M_{p l}=10^{18} \mathrm{GeV}$

\begin{tabular}{|c|c|c|c|c|c|c|c|c|c|}
\hline \multirow{2}{*}{$\begin{array}{l}V_{0} \\
\lambda\end{array}$} & \multicolumn{3}{|c|}{$10^{-16} M_{p l}^{4}$} & \multicolumn{3}{|c|}{$10^{-17} M_{p l}^{4}$} & \multicolumn{3}{|c|}{$10^{-18} M_{p l}^{4}$} \\
\hline & \multicolumn{3}{|c|}{$5.65 \cdot 10^{-6}$} & \multicolumn{3}{|c|}{$5.65 \cdot 10^{-7}$} & \multicolumn{3}{|c|}{$5.65 \cdot 10^{-8}$} \\
\hline$N$ & 50 & 60 & 70 & 50 & 60 & 70 & 50 & 60 & 70 \\
\hline$r$ & 0.0341 & 0.0263 & 0.0209 & 0.0475 & 0.0387 & 0.0325 & 0.0540 & 0.0447 & 0.0382 \\
\hline $\begin{array}{l}n_{s} \\
\alpha=\end{array}$ & \multicolumn{9}{|c|}{$n=4, \quad V_{0}=10^{-16} M_{p l}^{4}$} \\
\hline$M$ & \multicolumn{3}{|l|}{$10^{-6}$} & \multicolumn{3}{|l|}{$5 \cdot 10^{-7}$} & \multicolumn{3}{|l|}{$10^{-7}$} \\
\hline$\lambda$ & \multicolumn{3}{|c|}{$5.65 \cdot 10^{-6}$} & \multicolumn{3}{|c|}{$1.13 \cdot 10^{-5}$} & \multicolumn{3}{|c|}{$5.65 \cdot 10^{-5}$} \\
\hline$N$ & 50 & 60 & 70 & 50 & 60 & 70 & 50 & 60 & 70 \\
\hline$r$ & 0.0341 & 0.0263 & 0.0209 & 0.0415 & 0.0332 & 0.0273 & 0.0504 & 0.0414 & 0.0351 \\
\hline$n_{s}$ & 0.9649 & 0.9700 & 0.9736 & 0.9667 & 0.9720 & 0.9757 & 0.9678 & 0.9731 & 0.9769 \\
\hline
\end{tabular}




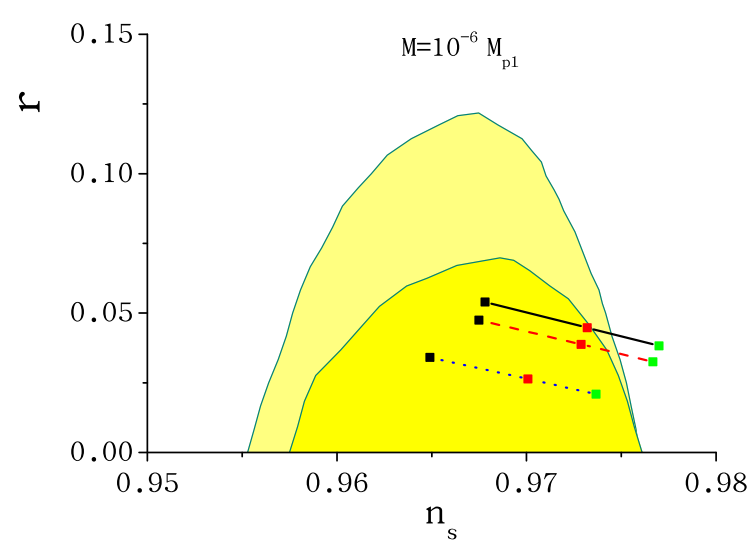

Fig. $31 \sigma$ (yellow) and $2 \sigma$ (light yellow) contours for Planck 2018 results (Planck $+T T+$ low $P$ ) [78], on the $n_{\mathrm{s}}-r$ plane. Moreover, we depict the predictions of Table 2, of the scenario at hand for fixed $\alpha=2, n=4$. In every line the first (black) point corresponds to efolding number $N=50$, the middle (red) point to $N=60$, and the third (green) to $N=70$. Left panel: Fixed $M=10^{-6} M_{p l}$. Black-

\section{Conclusions}

In this work we revisited inflation with non-canonical scalar fields by applying deformed-steepness exponential potentials. Non-canonical kinetic terms can arise straightforwardly in models of supergravity and superstrings, while exponential potentials have remarkable properties, as they greatly facilitate slow roll and result to scaling behaviour at large scales.

As we have shown, the resulting scenario can lead to inflationary observables, and in particular to scalar spectral index of the curvature perturbations $n_{\mathrm{S}}$ and tensor-to-scalar ratio $r$, in remarkable agreement with the observations of Planck 2018 , being well inside the $1 \sigma$ region. Apart from observational predictability, a significant additional advantage of the proposed scenario arises from the theoretical point of view. In particular, in order to obtain acceptable observables, in simple non-canonical models one needs to use relatively large non-canonical exponent $\alpha$ or ranges of values for the non-canonicality scale $M$, while in canonical models with deformed-steepness potentials relatively large values of the extra exponent $n$ need to be imposed, and, hence, these models cannot be well-justified theoretically. On the other hand, in the scenario of the present work the exponents $\alpha$ and $n$ are small, as well as the non-canonicality and potential scales $M$ and $V_{0}$ remain in reasonable sub-Planckian regions.

We mention here that the motivation for the deformedsteepness parameter $n$ of [63] was the improved inflationary observables (specifically $r$ ) compared to the simplest slowroll models. Moreover, the motivation for the extra parameter $\alpha$ compared to canonical models is to also improve $r$. Nevertheless, in works with non-minimal kinetic terms but with no deformed-steepness parameter $(n=1)$ the improvement

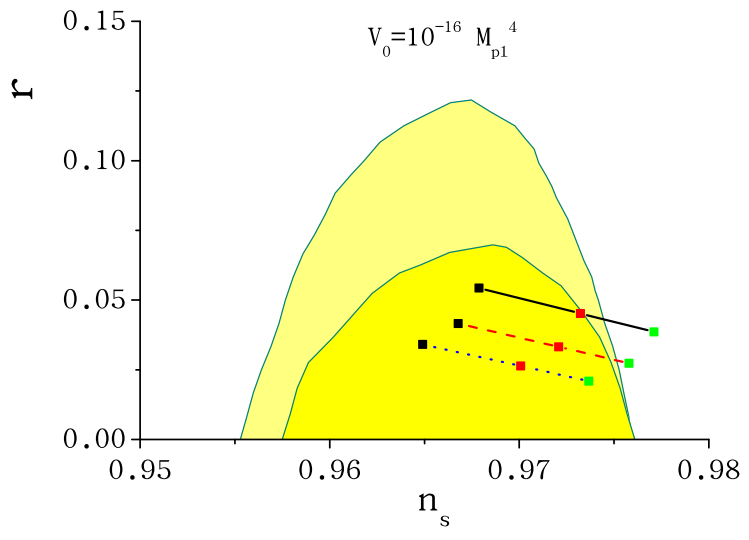

solid for $V_{0}=10^{-18} M_{p l}^{4}$, red-dashed for $V_{0}=10^{-17} M_{p l}^{4}$, blue-dotted for $V_{0}=10^{-16} M_{p l}^{4}$. Right panel: Fixed $V_{0}=10^{-16} M_{p l}^{4}$. Black-solid for $M=10^{-7} M_{p l}$, red-dashed for $M=5 \cdot 10^{-7} M_{p l}$, red-dotted for $M=10^{-6} M_{p l}$. In all cases the value of $\lambda$ is determined through the observational constraint (2.22), while $M_{p l}=10^{18} \mathrm{GeV}$

of the observables is obtained for very large $\alpha$ values (of the order of 100 or 1000 [36]). Hence, the motivation for allowing both $n \neq 1$ and $\alpha \neq 1$ is to be able to obtain improved observables, but with very low, and thus closer to natural, $\alpha$. Although the scenario has extra parameters, the improvement in observables (smaller tensor-to-scalar ratio values), as well as the higher naturalness is worth the price.

Our analysis revealed that, for a given $\alpha$, as $n$ increases both $n_{\mathrm{s}}$ and $r$ decrease too, while on the other hand, for a given $n$, as $\alpha$ increases there is no particular tendency for $n_{\mathrm{s}}$ and $r$. Additionally, for fixed $M$, increasing $V_{0}$ leads to lower values of $r$ and $n_{\mathrm{s}}$, while for fixed $V_{0}$, increasing $M$ leads to lower values of $r$ and $n_{\mathrm{s}}$ too.

In summary, we showed that revisiting non-canonical inflation models by applying potentials with deformed steepness, increases the observational predictability, bringing the scalar spectral index and the tensor-to-scalar ratio more deeply into the observational contours, offering a better theoretical justification for the required parameters. This combination of observational efficiency and theoretical justification is a significant advantage of the scenario at hand, and hence, non-canonical models with deformed-steepness potential need to be further explored, as additional observational data will be coming forward.

Data Availability Statement This manuscript has no associated data or the data will not be deposited. [Authors' comment: The work is mainly theoretical in nature and so does not involve any data or data analysis.]

Open Access This article is licensed under a Creative Commons Attribution 4.0 International License, which permits use, sharing, adaptation, distribution and reproduction in any medium or format, as long as you give appropriate credit to the original author(s) and the source, provide a link to the Creative Commons licence, and indicate if changes were made. The images or other third party material in this article 
are included in the article's Creative Commons licence, unless indicated otherwise in a credit line to the material. If material is not included in the article's Creative Commons licence and your intended use is not permitted by statutory regulation or exceeds the permitted use, you will need to obtain permission directly from the copyright holder. To view a copy of this licence, visit http://creativecomm ons.org/licenses/by/4.0/.

Funded by SCOAP ${ }^{3}$.

\section{References}

1. A.A. Starobinsky, A new type of isotropic cosmological models without singularity. Phys. Lett. B 91, 99 (1980)

2. D. Kazanas, Dynamics of the universe and spontaneous symmetry breaking. Astrophys. J. Lett. 241, L59-L63 (1980)

3. K. Sato, First order phase transition of a vacuum and expansion of the universe. Mon. Not. R. Astron. Soc. 195, 467 (1981)

4. A.H. Guth, The inflationary universe: a possible solution to the horizon and flatness problems. Phys. Rev. D 23, 347 (1981)

5. A.D. Linde, A new inflationary universe scenario: a possible solution of the horizon, flatness, homogeneity, isotropy and primordial monopole problems. Phys. Lett. B 108, 389 (1982)

6. K.A. Olive, Inflation. Phys. Rept. 190, 307 (1990)

7. D.H. Lyth, A. Riotto, Particle physics models of inflation and the cosmological density perturbation. Phys. Rept. 314, 1 (1999). arXiv:hep-ph/9807278

8. N. Bartolo, E. Komatsu, S. Matarrese, A. Riotto, Non-Gaussianity from inflation: theory and observations. Phys. Rept. 402, 103 (2004). arXiv:astro-ph/0406398

9. S. Nojiri, S.D. Odintsov, Unified cosmic history in modified gravity: from $\mathrm{F}(\mathrm{R})$ theory to Lorentz non-invariant models. Phys. Rept. 505, 59 (2011). arXiv: 1011.0544

10. J. Martin, C. Ringeval, V. Vennin, Encyclopaedia inflationaris. Phys. Dark Univ. 5-6, 75-235 (2014). arXiv:1303.3787

11. S.A. Appleby, R.A. Battye, A.A. Starobinsky, Curing singularities in cosmological evolution of $\mathrm{F}(\mathrm{R})$ gravity. JCAP 06, 005 (2010). arXiv:0909.1737 [astro-ph.CO]

12. S. Nojiri, S.D. Odintsov, Modified gravity with negative and positive powers of the curvature: Unification of the inflation and of the cosmic acceleration. Phys. Rev. D 68, 123512 (2003). arXiv:hep-th/030728

13. B.M. Carter, I.P. Neupane, Towards inflation and dark energy cosmologies from modified Gauss-Bonnet theory. JCAP 06, 004 (2006). arXiv:hep-th/0512262

14. R. Ferraro, F. Fiorini, Modified teleparallel gravity: inflation without inflaton. Phys. Rev. D 75, 084031 (2007). arXiv:gr-qc/0610067

15. C. Germani, A. Kehagias, New model of inflation with nonminimal derivative coupling of standard model Higgs Boson to gravity. Phys. Rev. Lett. 105, 011302 (2010). arXiv:1003.2635

16. L. Sebastiani, G. Cognola, R. Myrzakulov, S. Odintsov, S. Zerbini, Nearly Starobinsky inflation from modified gravity. Phys. Rev. D 89(2), 023518 (2014). arXiv:1311.0744

17. J.R. Ellis, D.V. Nanopoulos, K.A. Olive, K. Tamvakis, Cosmological inflation cries out for supersymmetry. Phys. Lett. B 118, 335-339 (1982)

18. J.R. Ellis, A.D. Linde, D.V. Nanopoulos, Inflation can save the gravitation. Phys. Lett. B 118, 59-64 (1982)

19. G.R. Dvali, Q. Shafi, R.K. Schaefer, Large scale structure and supersymmetric inflation without fine tuning. Phys. Rev. Lett. 73, 1886-1889 (1994). arXiv:hep-ph/9406319

20. G.G. Ross, S. Sarkar, Successful supersymmetric inflation. Nucl. Phys. B 461, 597-624 (1996). arXiv:hep-ph/9506283
21. D.V. Nanopoulos, K.A. Olive, M. Srednicki, K. Tamvakis, Primordial inflation in simple supergravity. Phys. Lett. B 123, 41-44 (1983)

22. D. Ewan, Stewart, inflation, supergravity and superstrings. Phys. Rev. D 51, 6847-6853 (1995). arXiv:hep-ph/9405389

23. E. Halyo, Hybrid inflation from supergravity D-terms. Phys. Lett. B 387, $43-47$ (1996). arXiv:hep-ph/9606423

24. D. Andrei, A. Linde, R. Antonio, Hybrid inflation in supergravity. Phys. Rev. D 56, 1841-1844 (1997). arXiv:hep-ph/9703209

25. Q. Shafi, C. Wetterich, Inflation from higher dimensions. Nucl. Phys. B 289, 787-809 (1987)

26. C. Ignatios Antoniadis, J.R. Bachas, D.V. Ellis, Nanopoulos, cosmological string theories and discrete inflation. Phys. Lett. B 211, 393-399 (1988)

27. G.R. Dvali, S.H.H. Tye, Brane inflation. Phys. Lett. B 450, 72-82 (1999). arXiv:hep-ph/9812483

28. F.C. Adams et al., Natural inflation: particle physics models, power law spectra for large scale structure, and constraints from COBE. Phys. Lett. D 47, 426-455 (1993). arXiv:hep-ph/9207245

29. C. Armendariz-Picon, T. Damour, V.F. Mukhanov, k-inflation. Phys. Lett. B 458, 209-218 (1999). arXiv:hep-th/9904075

30. J. Garriga, V.F. Mukhanov, Perturbations in k-inflation. Phys. Lett. B 458, 219-225 (1999). arXiv:hep-th/9904176

31. V.F. Mukhanov, A. Vikman, Enhancing the tensor-to-scalar ratio in simple inflation. JCAP 02, 004 (2006). arXiv:astro-ph/0512066

32. G. Barenboim, W.H. Kinney, Slow roll in simple non-canonical inflation. JCAP 03, 014 (2007). arXiv:astro-ph/0701343

33. K. Tzirakis, W.H. Kinney, Non-canonical generalizations of slowroll inflation models. JCAP 01, 028 (2009). arXiv:0810.0270

34. P. Franche, R. Gwyn, B. Underwood, A. Wissanji, Attractive Lagrangians for non-canonical inflation. Phys. Rev. D 81, 123526 (2010). arXiv:0912.1857

35. P. Franche, R. Gwyn, B. Underwood, A. Wissanji, Initial conditions for non-canonical inflation. Phys. Rev. D 82, 063528 (2010). arXiv: 1002.2639

36. S. Unnikrishnan, V. Sahni, A. Toporensky, Refining inflation using non-canonical scalars. JCAP 08, 018 (2012). arXiv:1205.0786

37. R. Gwyn, M. Rummel, A. Westphal, Relations between canonical and non-canonical inflation. JCAP 12, 010 (2013). arXiv: 1212.4135

38. D.A. Easson, B.A. Powell, The degeneracy problem in noncanonical inflation. JCAP 03, 028 (2013). arXiv: 1212.4154

39. X.M. Zhang, J.Y. Zhu, Extension of warm inflation to noncanonical scalar fields. Phys. Rev. D 90(12), 123519 (2014). arXiv:1402.0205

40. R. Gwyn, J.L. Lehners, Non-canonical inflation in supergravity. JHEP 05, 050 (2014). arXiv:1402.5120

41. M.W. Hossain, R. Myrzakulov, M. Sami, E.N. Saridakis, Variable gravity: a suitable framework for quintessential inflation. Phys. Rev. D 90(2), 023512 (2014). arXiv:1402.6661

42. K. Rezazadeh, K. Karami, P. Karimi, Intermediate inflation from a non-canonical scalar field. JCAP 09, 053 (2015). arXiv:1411.7302

43. S. Cespedes, A.C. Davis, Non-canonical inflation coupled to matter. JCAP 11, 014 (2015). arXiv:1506.01244

44. N.K. Stein, W.H. Kinney, Planck limits on non-canonical generalizations of large-field inflation models. JCAP 04, 006 (2017). arXiv: 1609.08959

45. K. Dimopoulos, C. Owen, Quintessential Inflation with $\alpha$ attractors. JCAP 06, 027 (2017). arXiv: 1703.00305

46. A. Mohammadi, K. Saaidi, H. Sheikhahmadi, Constant-roll approach to non-canonical inflation. Phys. Rev. D 100(8), 083520 (2019). arXiv: 1803.01715

47. M. Naderi, A. Aghamohammadi, A. Refaei, H. Sheikhahmadi, Intermediate inflation with non-canonical scalar field in the low anisotropy Universe. Mod. Phys. Lett. A 34(33), 1950272 (2019). arXiv: 1809.02348 
48. A.Y. Kamenshchik, A. Tronconi, T. Vardanyan, G. Venturi, Noncanonical inflation and primordial black holes production. Phys. Lett. B 791, 201-205 (2019). arXiv:1812.02547]

49. T.Q. Do, W. Kao, I.C. Lin, CMB imprints of non-canonical anisotropic inflation (2020). arXiv:2003.04266

50. N. Bose, A. Majumdar, A k-essence model of inflation, dark matter and dark energy. Phys. Rev. D 79, 103517 (2009). arXiv:0812.4131

51. J. De-Santiago, J.L. Cervantes-Cota, Generalizing a unified model of dark matter, dark energy, and inflation with non canonical kinetic term. Phys. Rev. D 83, 063502 (2011). arXiv:1102.1777

52. V. Sahni, A.A. Sen, A new recipe for $\Lambda$ CDM. Eur. Phys. J. C 77(4), 225 (2017). arXiv: 1510.09010

53. S.S. Mishra, V. Sahni, Unifying dark matter and dark energy with non-canonical scalars (2018). arXiv:1803.09767

54. T. Kobayashi, M. Yamaguchi, J. Yokoyama, G-inflation, Inflation driven by the Galileon field. Phys. Rev. Lett. 105, 231302 (2010). arXiv: 1008.0603

55. C. Burrage, C. de Rham, D. Seery, A.J. Tolley, Galileon inflation. JCAP 01, 014 (2011). arXiv:1009.2497

56. J. Ohashi, S. Tsujikawa, Potential-driven Galileon inflation. JCAP 10, 035 (2012). arXiv:1207.4879

57. S. Tsujikawa, The effective field theory of inflation/dark energy and the Horndeski theory. Lect. Notes Phys. 892, 97-136 (2015). arXiv: 1404.2684

58. A.A. Starobinsky, S.V. Sushkov, M.S. Volkov, The screening Horndeski cosmologies. JCAP 06, 007 (2016). arXiv:1604.06085

59. L. Sebastiani, S. Myrzakul, R. Myrzakulov, Reconstruction of kessence inflation in Horndeski gravity. Eur. Phys. J. Plus 132(10), 433 (2017). arXiv: 1702.00064

60. G. Koutsoumbas, K. Ntrekis, E. Papantonopoulos, E.N. Saridakis, Unification of dark matter - dark energy in generalized Galileon theories. JCAP 02, 003 (2018). arXiv:1704.08640]

61. C.Q. Geng, M.W. Hossain, R. Myrzakulov, M. Sami, E.N. Saridakis, Quintessential inflation with canonical and noncanonical scalar fields and Planck 2015 results. Phys. Rev. D 92(2), 023522 (2015). arXiv:1502.03597

62. K. Rezazadeh, K. Karami, S. Hashemi, Tachyon inflation with steep potentials. Phys. Rev. D 95(10), 103506 (2017). arXiv: 1508.04760

63. C.Q. Geng, C.C. Lee, M. Sami, E.N. Saridakis, A.A. Starobinsky, Observational constraints on successful model of quintessential Inflation. JCAP 06, 011 (2017). arXiv: 1705.01329

64. S. Ahmad, R. Myrzakulo, M. Sami, Relic gravitational waves from Quintessential Inflation. Phys. Rev. D 96(6), 063515 (2017). arXiv: 1705.02133
65. A. Agarwal, R. Myrzakulov, M. Sami, N.K. Singh, Quintessential inflation in a thawing realization. Phys. Lett. B 770, 200-208 (2017). arXiv: 1708.00156

66. M. Skugoreva, M. Sami, N. Jaman, Emergence of cosmological scaling behavior in the asymptotic regime. Phys. Rev. D 100(4), 043512 (2019). arXiv: 1901.06036

67. S. Das, M. Banerjee, N. Roy, Dynamical system analysis for steep potentials. JCAP 08, 024 (2019). arXiv: 1903.02288

68. G.B. Lima, R.O. Ramos, Unified early and late Universe cosmology through dissipative effects in steep quintessential inflation potential models. Phys. Rev. D 100(12), 123529 (2019). arXiv:1910.05185

69. S. Nojiri, S.D. Odintsov, V.K. Oikonomou, Modified gravity theories on a Nutshell: inflation, bounce and late-time evolution. Phys. Rept. 692, 1-104 (2017). arXiv:1705.11098

70. S. Bhattacharya, M.R. Gangopadhyay, Study in the noncanonical domain of Goldstone inflation. Phys. Rev. D 101(2), 023509 (2020). arXiv:1812.08141

71. S. Bhattacharya, M.R. Gangopadhyay, I. Banerjee, S. Chakraborty, S. SenGupta, Phys. Rev. D 99(2), 023515, (2019). arXiv: 1806.11327

72. S. Li, A.R. Liddle, Observational constraints on K-inflation models. JCAP 10, 011 (2012). arXiv:1204.6214

73. S. Unnikrishnan, Can cosmological observations uniquely determine the nature of dark energy? Phys. Rev. D 78, 063007 (2008). arXiv:0805.0578

74. J.E. Lidsey, A.R. Liddle, E.W. Kolb, E.J. Copeland, T. Barreiro, M. Abney, Reconstructing the inflation potential: an overview. Rev. Mod. Phys. 69, 373 (1997). arXiv:astro-ph/9508078

75. D.I. Kaiser, Primordial spectral indices from generalized Einstein theories. Phys. Rev. D 52, 4295 (1995). arXiv:astro-ph/9408044

76. M. Sasaki, E.D. Stewart, A General analytic formula for the spectral index of the density perturbations produced during inflation. Prog. Theor. Phys. 95, 71 (1996). arXiv:astro-ph/9507001

77. R.P. Woodard, Perturbative quantum gravity comes of age. Int. J. Mod. Phys. D 23(09), 1430020 (2014). arXiv:1407.4748

78. Y. Akrami et al. (Planck Collaboration), Planck 2018 results. X. Constraints on inflation (2018). arXiv:1807.06211]

79. G. German, A. de la Macorra, M. Mondragon, Low scale supergravity inflation with R symmetry. Phys. Lett. B 494, 311-317 (2000). arXiv:hep-th/0006200 